\title{
Perspective review on applications of optics in spinal surgery
}

Daipayan Guha

Victor X. D. Yang 


\title{
Perspective review on applications of optics in spinal surgery
}

\author{
Daipayan Guha ${ }^{a}$ and Victor X. D. Yang ${ }^{a, b, c, *}$ \\ aUniversity of Toronto, Division of Neurosurgery, Toronto, Ontario, Canada \\ bSunnybrook Health Sciences Centre, Toronto, Ontario, Canada \\ 'Ryerson University, Bioengineering and Biophotonics Laboratory, Toronto, Ontario, Canada
}

\begin{abstract}
Optical technologies may be applied to multiple facets of spinal surgery from diagnostics to intraoperative image guidance to therapeutics. In diagnostics, the current standard remains cross-sectional static imaging. Optical surface scanning tools may have an important role; however, significant work is required to clearly correlate surface metrics to radiographic and clinically relevant spinal anatomy and alignment. In the realm of intraoperative image guidance, optical tracking is widely developed as the current standard of instrument tracking, however remains compromised by line-of-sight issues and more globally cumbersome registration workflows. Surface scanning registration tools are being refined to address concerns over workflow and learning curves, and allow real-time update of tissue deformation; however, the line-of-sight issues plaguing instrument tracking remain to be addressed. In therapeutics, optical applications exist in both visualization, in the form of endoscopes, and ablation, in the form of lasers. Further work is required to extend the feasibility of laser ablation to multiple tissues, including disc, bone, and tumor, in a safe and time-efficient manner. Finally, we postulate some of the short- and long-term opportunities for future growth of optical techniques in the context of spinal surgery. Particular emphasis is placed on intraoperative image guidance, the area of the authors' primary expertise. (C) 2018 Society of Photo-Optical Instrumentation Engineers (SPIE) [DOI: 10.1117/1.JBO.23.6.060601]
\end{abstract}

Keywords: optics; photonics; laser; spine surgery; image guidance.

Paper 180200-PER received Apr. 4, 2018; accepted for publication May 23, 2018; published online Jun. 11, 2018.

\section{Introduction}

The surgical treatment of spinal disorders represents a combination of musculoskeletal (MSK) and neurological elements, performed therefore by both orthopedic surgeons and neurosurgeons in varying practice patterns. The care pathway of a patient with spinal pathology begins with evaluation clinically and radiographically. Once a decision has been made for surgical treatment, the therapeutic intervention is often assisted by intraoperative image guidance. In each of these elements (diagnostics, therapeutics, and image-guidance), optical technologies currently play a significant role, with numerous possibilities for further development in the near and distant future. In this paper, we outline our perspectives on the current challenges faced by clinicians in each of the diagnostic and intraoperative phases of spinal surgery, existing optical approaches in these phases, and opportunities for further development.

\section{Diagnostics}

\subsection{Current Paradigm and Challenges}

The initial and preoperative evaluation of the spinal patient involves a thorough clinical examination in conjunction with diagnostic imaging. Depending on whether the presenting symptoms are largely MSK or neurological in nature, imaging may involve any combination of plain X-rays (XR), computed tomography (CT) imaging, or magnetic resonance (MR) imaging. Osseous structures are best visualized on XR or CT, with their associated radiation burden, while neural elements and soft-tissue structures are best seen on MRI.

In the current paradigm, all diagnostic imaging modalities represent a static snapshot, taken with the patient in a position that may not replicate a clinically relevant scenario. For instance, in a patient with clinical signs of cervical myelopathy as a result of dynamic cervical cord compression, a standard MRI taken with the patient supine may not demonstrate significant cord compression that typically occurs with the patient standing up or with their neck flexed, with additional load placed on the ligaments connecting cervical vertebrae. ${ }^{1,2}$ In patients with scoliosis, radiographic Cobb angles measuring intervertebral alignment on XR may not correlate with the external rib/torso deformity, which may be more socially debilitating for patients, and the extent of radiographic correction postoperatively similarly does not necessarily correlate linearly to external improvement. ${ }^{3}$ Variations in each imaging modality are currently applied to explore specific pathologies more realistically. For instance, flexion-extension lateral XR views may be employed to assess sagittal-plane dynamic instability that may require instrumentation for internal stabilization. Three-foot standing XR is often obtained to assess global spinal alignment in evaluating patients, both adolescent and adult, with significant spinal deformity. Standing MRI or supine MRI with axial loading, while not mainstays of diagnostic imaging, has been applied to multiple scenarios. ${ }^{4-6}$ 
A second limitation specific to XR and CT imaging modalities pertains to their cumulative burden of ionizing radiation, of significance particularly in the adolescent scoliosis population. These patients often receive multiple scans as part of their workup to evaluate progression over time, as well as intraoperatively and postoperatively in follow-up, for a significant cumulative radiation burden. ${ }^{7}$ This may be associated with a greater lifetime risk of cancer; a higher lifetime incidence of breast cancer has been demonstrated in women with scoliosis. ${ }^{8-10}$ Low-dose protocols have been investigated in comparative studies of anatomic adequacy relative to standard-dose protocols, with substantial progress made in lowering the cumulative radiation burden particularly for adolescent patients. ${ }^{11,12}$

\subsection{Current Optical Applications}

The limitations of current cross-sectional imaging, with respect to lack of dynamic information as well as associated radiation burden, may be partially overcome with radiationless optical techniques. In the context of adolescent scoliosis, surface topography analysis has been applied over the past two decades to assess external deformity in a standing upright posture. Optical scanning techniques applied for surface topography in this context include moiré-fringe mapping, ${ }^{13}$ structured-light imaging, ${ }^{14-17}$ ultrasound and laser range-scanning. ${ }^{18}$ While radiographic Cobb angles, that is, the angles between two given vertebral endplates on XR/CT, are typically taken as the gold standard in the evaluation of spinal deformity, they assess deformity only in the plane in which they are measured rather than as a global indicator of alignment, and have demonstrated poor correlation with back surface metrics. ${ }^{19-21}$ Nonetheless, a number of commercial surface-scanning systems are currently in common usage in the evaluation and ongoing follow-up of scoliosis patients, with utility in assessing temporal trends, if less so in dictating radiographic outcome. ${ }^{14}$

\subsection{Future Directions}

To date, however, the surface-scanning techniques commonplace in the context of adolescent scoliosis have not been applied to evaluating other populations or pathologies. A large contributor to this is, as discussed, poor correlation between radiographic metrics commonly used by spinal surgeons to evaluate, plan, and execute deformity-correcting procedures, and back surface parameters.

The first step in extending optical applications to the diagnosis of multiple spinal pathologies is therefore a need for large epidemiological studies correlating torso and back surface topographic parameters to radiographic metrics or measures of global spinal alignment. In the context of spinal diagnostics, surface-scanning techniques are the most mature optical technology currently available, hence extension of its applications in the short-term is predicated on future epidemiological work rather than additional technical development. Epidemiological work may be performed, for instance, to extend the utility of surface-scanning metrics from adolescent scoliosis first to adult degenerative deformities, which tend to be more focal and therefore present less external evidence of spinal malalignment. This application will, however, require significantly greater spatial resolution to account for the more focal pathology. Given that existing surface-scanning techniques have been poorly correlated to radiologic metrics of spinal alignment, longer-term advances in noninvasive optical diagnostics will likely require altogether technologies able to provide crosssectional imaging in real-time with resolution equivalent to that of modern MR imaging.

\section{Intraoperative Image Guidance}

\subsection{Current Paradigm and Challenges}

Computer-assisted navigation (CAN) for intraoperative image guidance was pioneered initially for the localization of subsurface structures in cranial neurosurgery. It has subsequently been applied to spinal procedures, initially for the guidance of lumbar pedicle screws. ${ }^{22}$ Instrumentation guidance remains the primary application for CAN by most spinal surgeons, with multiple systematic reviews and meta-analyses reporting on the radiographic accuracy of pedicle screws in the cervical, thoracic, and lumbosacral spine, and in multiple clinical contexts including minimally invasive (MIS) percutaneous instrumentation as well as in adolescent idiopathic scoliosis patients. ${ }^{23-32}$ CAN has been proven to reduce radiographic pedicle screw breach rates to under $10 \%$ in the cervical spine, under $7 \%$ in the thoracic spine, and under $5 \%$ in the lumbar spine. ${ }^{27}$

Contemporary spinal CAN systems register predominantly to either preoperative $\mathrm{CT}$ imaging or intraoperative conebeam CT (CBCT) images acquired via isocentric threedimensional (3-D) fluoroscopy or 360-deg CBCT scanning. Regardless of technique, CAN usage has been shown to improve the radiographic accuracy of pedicle screw placement across all levels of the spine. ${ }^{23,27} \mathrm{~A}$ multitude of subsequent studies has demonstrated reduced occupational radiation dose, i.e., to OR personnel, with 3-D fluoroscopy-based navigation, ${ }^{33-35}$ as well as with intra-operative CBCT. ${ }^{36-38}$ However, while CAN reduces the radiation exposure to surgical and OR personnel, it does appear that this is more a result of shifting the burden of radiation to the patient rather than a reduction in overall radiation exposure. Lange et al. ${ }^{39}$ have estimated that three or more intraoperative $\mathrm{O}$-arm imaging cycles, at standard manufacturerrecommended dosing, results in patient radiation exposure equivalent to that one of standard abdominal CT scan. Therefore, while CAN techniques may reduce occupational radiation exposure for OR personnel, particularly in traditionally fluoroscopy-heavy procedures including MIS and deformity corrections, the burden of radiation exposure remains, and in the current paradigm of CAN techniques is shifted to the patient rather than eliminated entirely. ${ }^{38}$

Adoption of CAN by spinal surgeons is further limited by concerns over temporal efficiency, particularly as a result of cumbersome registration workflow. ${ }^{40}$ In a comparative study of O-arm (3-D CBCT) versus fluoroscopy guidance for MIS lateral interbody lumbar fusions, Zhang et al. ${ }^{41}$ demonstrated a statistically insignificant increase in operative time with CAN guidance. In larger in vivo studies, both Rajasekaran et al. ${ }^{42}$ and Tabaraee et al. $^{43}$ found time-equivalence for 3-D CBCT-based navigation versus fluoroscopy for the placement of posterior thoracolumbar pedicle screws. While a temporal efficiency benefit to CAN has yet to be demonstrated with current paradigms of navigation, there does appear to be a significant learning curve, with increased operative times early in the curve followed by time-equivalence or even modest savings once sufficient familiarity has been achieved. $^{44}$ 


\subsection{Current Optical Applications}

Current optical applications for intraoperative CAN in spinal surgery are largely in the realm of instrument tracking. Modern CAN systems track instrumentation either using electromagnetic tracking, with a small coil embedded in the tip of the instrument to be tracked, used almost exclusively for craniofacial and orthopedic applications, as well as optically. Optical tracking systems (OTS) employ an infrared camera \pm emitter mounted on a mobile platform to track instruments and reference frames either passively, with the use of IR-reflective spheres, or actively, with IR-LED emitters on the instruments themselves. There has been some suggestion, particularly among early-generation technologies, that active OTS provides lower tracking error and greater consistency than passive systems; Khadem et al. ${ }^{45}$ compared both active and passive OTS units from the same manufacturer and found RMS jitter of $0.058 \pm 0.037 \mathrm{~mm}$ with active OTS and $0.115 \pm 0.075 \mathrm{~mm}$ with passive. These differences have been mitigated in modern devices; current optical systems are able to track individual markers with an accuracy of $\sim 0.25 \mathrm{~mm}$, and instrument tips at accuracies of 1 to $2 \mathrm{~mm} .{ }^{46}$ However, OTS tool tracking relies on a precalibrated relationship between the IR tracker array mounted on an instrument and the instrument tip, hence is unable to track needles and other nonrigid tools. More importantly, accurate and real-time optical tracking necessitates constant line-of-sight between the IR camera \pm emitter platform, and the reference frame as well as the tracking array on each monitored instrument. Clutter in the surgical field, and even the physical position of OR personnel and the hand position of the tool operator, can greatly influence optical instrument tracking. Moreover, the relative positioning of the dynamic reference frame (DRF), tracker arrays on each tool, and the camera unit can influence the tracking error of the system; Khadem et al. demonstrated that the majority of OTS tracking error arises in the $z$-axis, that is, pointing directly away from the IR camera.
Finally, the number of tracker markers (reflective spheres or active LEDs) visible to the IR camera, as well as the distance from the tool tip to the centroid of its tracker array, also influence tracking error. ${ }^{46}$ It is also known that optical instrumenttracking accuracy in vivo degrades over time as well as with increasing distance from the DRF. ${ }^{47}$ Operator-dependent mechanisms to reduce optical tracking error therefore include situating the IR cameras as close to the surgical field as possible, and moreover aligning the camera $z$-axis with the direction in which clinical accuracy is least important, as well as ensuring that as many markers as possible are visible to the camera when affixing the DRF and tracking a given surgical instrument.

Optical techniques for navigation applications beyond instrument tracking are only recently beginning to emerge. Visibleband computer stereovision has been applied initially for the resolution of brain shift for updating cranial registrations, but has not yet gained clinical utility in spinal procedures. ${ }^{48}$ Prototype systems based on stereovision alone have been trialled in the context of spinal anatomy. ${ }^{49,50}$ Our group is the first to develop an optical active projection technique for patient-to-image registration based on structured-light illumination; the technology is now available as a commercial device from 7D Surgical Inc. ${ }^{\mathrm{TM}}$. In this technique, termed optical topographic imaging (OTI), the exposed surgical cavity is illuminated with a two-dimensional (2-D) spatially varying pattern of known periodicity and intensity, captured subsequently by grayscale visible-band cameras. The deformation of the projected structured-light pattern is then used to extract depth information and generate a 3 -D point cloud of the exposed surface (Fig. 1). This surface is then registered automatically to a segmented preoperatively acquired imaging dataset using surface-matching algorithms. ${ }^{51}$ Our research endeavors have demonstrated that this technique is significantly faster than existing paradigms of patient-to-image registration, with equivalent accuracy in each of the cervical and thoracolumbar spines. ${ }^{51}$

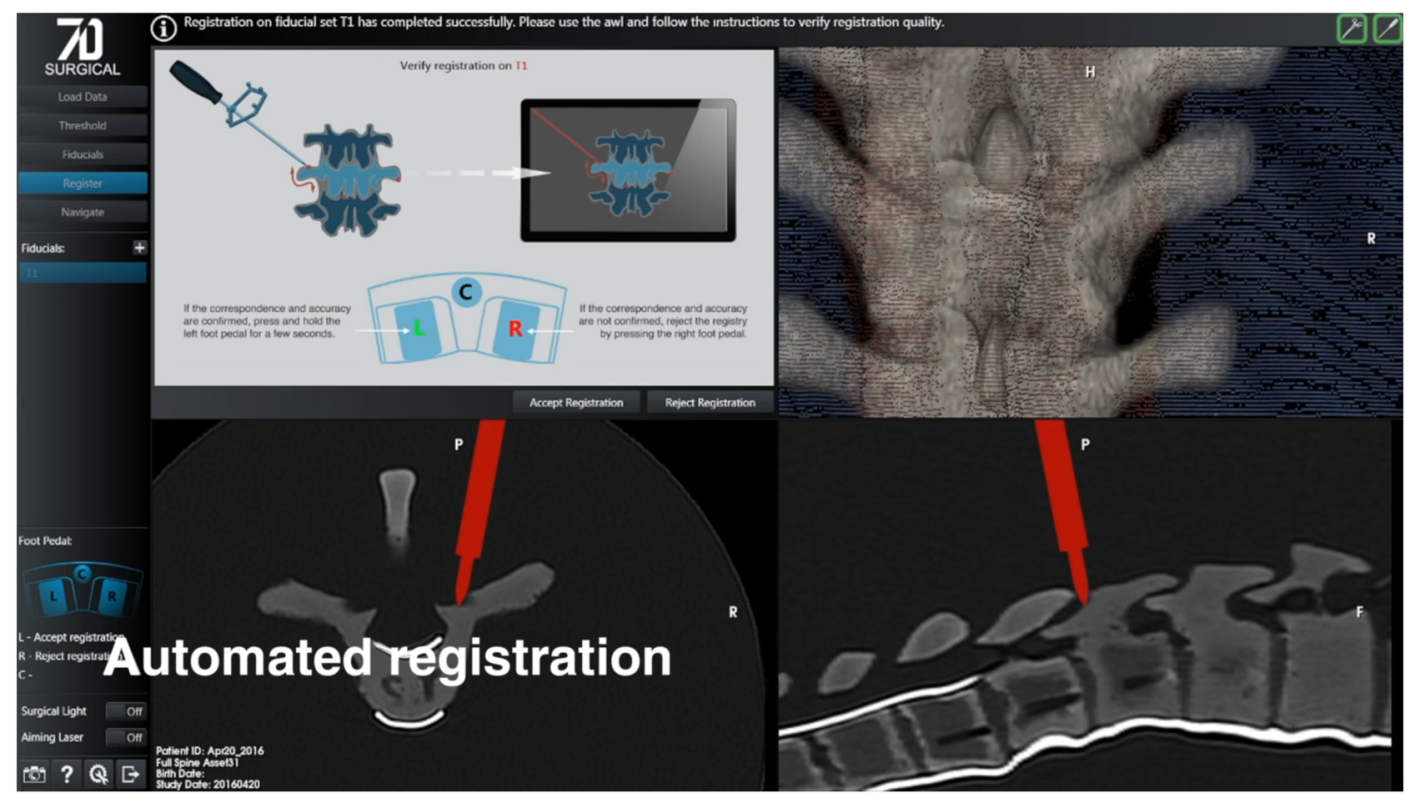

Fig. 1 Example of a user interface of an intraoperative navigation system based on OTI, developed by 7D Surgical Inc. 3-D point cloud of the posterior spine surface, generated via structured-light illumination, is shown in the top right panel, registered automatically to a 3-D reconstruction of the CT scan of the spine. Axial and sagittal reconstructions of the CT scan are shown in the bottom left and right panels, respectively. 


\subsection{Future Directions}

Given the relatively greater accuracy of optical relative to electromagnetic tracking systems, further development of optical instrument tracking is warranted. ${ }^{52}$ Future work in this arena should focus on alleviating the primary drawbacks of current IR tracking: line-of-sight issues and significant jitter along the axis of the cameras. With regards to the former, some manufacturers have already introduced tracker arrays, for both instruments and reference frames, with multiplanar configurations to minimize line-of-sight issues associated with misalignment of a single tracker-array plane with the camera longitudinal axis. Other approaches to the line-of-sight problem may also be feasible; for instance, one can imagine a scenario with repeater devices, either cameras or active LEDs, positioned at various locations throughout an operating room, such that instrument position may still be calculated accurately even if a specific set of LEDs or passive-reflective spheres are blocked by operating personnel or other equipment. This would be analogous to the repeaters and bridge modes applied in home/enterprise wireless networking to extend the range of a wireless network across a given space. For this technique to work, of course, the position of each repeater device relative to the central camera would have to be measured with high precision. Spatial 3-D accuracy of $<2 \mathrm{~mm}$ is typically accepted as the current industry expectation for intraoperative navigation techniques, though no definitive standard exists. Multiple groups have explored the feasibility of redundant optical tracking cameras to minimize occlusion and line-of-sight hindrances, with success in proof-of-concept but with limited translation to clinical settings due in part to high costs and cumbersome initial setup. ${ }^{53}$ However, with the advent of modern hybrid operating rooms in highly specialized centers, with built-in capabilities for biplanar fluoroscopy, 3-D imaging and navigation, coupled with the use of off-the-shelf camera components one can imagine improved adoption of redundant camera arrays.

With regard to optical applications for patient-to-image registration, several avenues of investigation may extend the capabilities of OTI or other machine-vision techniques. In the current paradigm of open spinal surgery, possibilities include the application of OTI for real-time continuous anatomical and instrument tracking, for machine learning-based adaptation to level localization, and for the scanning of nonosseous surfaces to eliminate the need for intraoperative imaging.

In all current paradigms of frameless stereotactic navigation, a DRF is required for relative instrument tracking and for maintenance of the initial registration. If the pose of exposed surgical anatomy could be tracked in real-time, however, this would permit updating the initial registration simultaneously. Similar principles have already been applied in the use of structuredlight illumination to update cranial registrations to compensate for intraoperative brain shift, albeit at noncontiguous intervals. ${ }^{48,54,55}$ With increases in the refresh rates of the visible-band cameras responsible for capturing the deformed structured-light illumination, and with appropriate computing power, an exposed surface could be tracked at a frequency on the order of 10 to $30 \mathrm{~Hz}$. More intriguingly, surgical tools within the detection range of stereocameras could also be tracked at the same frequency using the same visible-band cameras, using object identification algorithms to isolate and track a given surgical instrument. Modern infrared (IR) optical instrumenttracking systems update at a frequency of 20 to $60 \mathrm{~Hz}$, hence direct visible-band instrument tracking at a comparable frequency would not result in any appreciable lag to the operator. If computing power is insufficient to update registrations at this frequency, a hybrid system of IR tool tracking within the visible-band camera volume may also be considered, requiring an additional calibration to mesh the coordinate spaces of the IR and visible cameras. Either technique would eliminate the need for a DRF and thereby obviate one of the major pain points identified in current navigation workflows, as well as eliminate a significant source of instrument-tracking error. ${ }^{40,56}$

The ability of OTI to segment and track vertebral levels individually may also be applied to the real-time tracking of alignment intraoperatively. Particularly in long-segment deformity correction procedures, a common-use scenario for spinal CAN, the final desired alignment is decided a priori based on preoperative imaging parameters and the necessary operative maneuvers planned accordingly, i.e., what type of osteotomies may be required and at which levels to achieve the desired correction. Intraoperative confirmation of achievement of the desired alignment, however, remains a mainstay of traditional fluoroscopy, with repeated imaging required to iteratively confirm the required correction at each operated level in order to attain the desired global alignment. Real-time optical tracking of each segmental vertebral level allows independent registration to the corresponding imaging dataset; changes in intervertebral alignment may therefore be computed by repeat OTI registration, obviating the need for ongoing fluoroscopic imaging. Changes in global alignment may then be computed by summating the segmental changes in intervertebral alignment, to provide a radiationfree snapshot of global alignment. Particularly in the context of adolescent idiopathic scoliosis, extension of OTI to real-time segmental alignment tracking has the potential to significantly reduce radiation burden as well as time cost, major topics of ongoing investigation in the adolescent spine field. ${ }^{57,58}$

Another feasible application of vertebral imaging using OTI involves the localization of levels for surgical site identification. The target level to be operated on is typically identified using some combination of intraoperative localization, using fluoroscopy or mobile XR, and preoperative placement of radio-opaque markers which are then captured on preoperative imaging. ${ }^{59,60}$ Localization remains most challenging in the midthoracic region or with poor visibility on XR imaging, as levels must be counted from the occipitocervical junction (top-down) or lumbosacral junction (bottom-up). The rate of wrong-level surgery in the United States is $\sim 0.03 \%$ which, while small, remains unacceptably high for what should be a "never" incidence. ${ }^{61}$ With advances in machine learning algorithms for object recognition, ${ }^{62}$ as seen most evidently in the consumer electronics arena with applications such as Google Lens, an opportunity exists for a potential application of OTI in target level identification. A library of structured-light-based surface maps of various spinal levels may be constructed, with computation of relevant differentiating parameters such as laminar and spinous process dimensions, and incident angles of the lamina relative to cranial and caudal levels as well as to the spinous process; free parameters would also be available, to allow application of a standard neural network framework. Deep-learning algorithms may then be applied to identify a set of parameters reliably and uniquely identifying spinal levels, with testing and validation performed on a distinct set of scanned vertebrae. OTI imaging might then be applied to an open posterior exposure to identify the level without additional fluoroscopic imaging, or as a confirmatory measure if fluoroscopic identification is 
equivocal or difficult. This is likely most appropriate only in larger open exposures; in most minimally invasive approaches, the skin incision must be tailored to the target of interest, hence $\mathrm{XR}$ target localization prior to exposure remains a requirement. Certainly, this approach may be feasible, with precedent set by a similar algorithm employing a 2-D-3-D registration of intraoperative lateral XR to preoperative $\mathrm{CT}$ for automatic level confirmation, once levels had been reliably identified on the CT initially as the "gold standard." 63

Continuing along the established path of spinal surgery, OTI may also be applied to image other instruments in the operative field. A major limitation of OTI-CAN in its current form is the lack of integrated verification of instrumentation placement accuracy; automatically registered CAN techniques require an intraoperative imaging device, which may be used postinstrumentation to assess the accuracy of placed hardware and revise intraoperatively if necessary. A scenario can easily be envisioned, with minimal software modification, whereby structured-light illumination may be applied to scan a pedicle screw as it is partially inserted into a target cannulated tract. If the known screw diameter and length are specified, a virtual projection of the imaged screw may then be placed onto the concomitantly registered spine imaging, and automatically advanced into final position in the imaging space to provide a real-time "virtual" check on implant placement accuracy. The trajectory of pedicle screws is unlikely to change significantly after the screw has been partially threaded into the cannulated tract, particularly if the tract has been tapped previously, ${ }^{64}$ hence errors in the virtual projection can reasonably be assumed to be minimal. Instrumentation verification may therefore be performed in a radiation-free fashion, obviating the need for a bulky and costly intraoperative imaging device or postoperative CT, with its associated radiation burden.

Adaption of OTI for the scanning of soft-tissues in the context of spinal surgery is also within the realm of short-term possibility. Registration in its current prototype format is performed via scanning of rigid osseous anatomy, which, while readily accessible for open and even miniopen MIS approaches, precludes fully percutaneous approaches to the spine. Structured light-based 3-D scanning of the back has already been described in the context of noninvasive methods for evaluating patients with idiopathic scoliosis, ${ }^{65}$ using surface maps of the dorsal skin to compute metrics of axial deformity and global symmetry as surrogates for traditional radiographic alignment parameters. One can imagine performing a similar maneuver on a patient positioned prone on an operating table, or even coupling an OTI scanner to a motorized rotatory frame similar to those in isocentric fluoroscopy or CBCT devices, to generate a partially circumferential surface map of the dorsal skin anatomy. Applying a correction function to adjust for skin shift from supine preoperative imaging to prone operative positioning, registration may then theoretically be achieved using the skin surfaces alone, allowing image-guided fully percutaneous procedures. With these advancements, there would remain no limitations to OTI for spinal applications relative to current CAN techniques.

\section{Therapeutics}

\subsection{Current Paradigm and Challenges}

Spine surgical procedures typically involve the use of instruments for soft-tissue dissection, including electrocautery and various forceps and retractors, as well as osseous resection, including a combination of high-speed drills and rongeurs. Recent advances in MIS, image-guided, and robotically actuated spine surgery have allowed for smaller incisions and more targeted procedures; however, the end effectors remain those used in standard open procedures, perhaps with slight modifications to fit a tubular portal or robotic arm. Despite these modifications, the use of standard instruments through tubular portals or endoscopic approaches is technically challenging, associated with a steep learning curve, and is associated with poor visibility and dexterity when coupled with increasingly smaller surgical corridors. Moreover, the rigidity of these instruments, and requirement for direction visualization to avoid iatrogenic injury, often necessitates the creation of a wider surgical field to access deeper pathology. For instance, surgical decompression of ventral epidural metastatic tumor in the thoracic and high lumbar spine often requires resection of the facet joint complex on one side, to allow access to the ventral disease but therefore necessitating an instrumented fusion to maintain stability of the spinal column, adding potential morbidity and operative time. $^{66}$

The use of modern, high-speed drills and rongeurs for osseous resection, while efficacious and efficient, is nonetheless associated with some risk of injury to the underlying neural elements and coverings. The rate of dural tears is estimated to be $3.5 \%$ for primary microdiscectomy, and significantly greater at $8.5 \%$ for laminectomy for spinal stenosis, and $13.2 \%$ for revision microdiscectomy. ${ }^{67}$

\subsection{Current Optical Applications}

A number of optical approaches to in situ ablation are in current use, to address current limitations of bulky rigid instruments. Perhaps the most well studied is percutaneous laser discectomy (PLD), approved by the Food and Drug Administration in 1991 for the treatment of lumbar disc herniations. ${ }^{68}$ Initially, employing neodymium:yttrium-aluminum-garnet (YAG) and more recently holmium:YAG solid-state components, lasers are inserted percutaneously and guided fluoroscopically into the nucleus pulposus of the target disc, with injection of radiocontrast epidurally for confirmation of placement. Laser ablation of the nucleus pulposus is then performed, with a subsequent reduction in intradiscal pressure and therefore reduction of herniated fragments with decompression of the affected nerve root. This technique has been augmented subsequently with direct endoscopic visualization, termed percutaneous endoscopic laser discectomy (PELD). Few randomized controlled trials of PLD/PELD versus standard open microdiscectomy for lumbar radiculopathy have been performed, with the limited literature demonstrating noninferiority of PELD for patientreported pain and functional outcomes at 2-year follow-up; however, with a significantly greater rate of reoperation by 2- to 3-fold, and slower rate of recovery, in the PELD versus the open microdiscectomy cohort. ${ }^{69}$ Therefore, while PLD/PELD remains federally approved options for the treatment of refractory lumbar radiculopathy, they are not a mainstay of treatment particularly by spinal surgeons. Percutaneous laser ablation has been investigated in other spinal regions, for instance, for cervical disc decompression, with limited evidence of efficacy. ${ }^{70}$

Laser ablation has also been explored in the context of spinal oncology, specifically for epidural and vertebral-body metastatic disease. In patients with significant epidural cord compression from metastatic disease, open posterior surgical decompression 
is often undertaken to achieve "separation" of tumor from the spinal cord, to maximize the efficacy of subsequent radiation therapy. ${ }^{66}$ Above the midlumbar spine, however, ventral decompression from a posterior approach often necessitates lateral bony resection to allow ventral access without injuring the neural elements, subsequently requiring instrumentation for stabilization. MRI-guided percutaneous transpedicular insertion of a fiber diode laser has been described to target ventral epidural and vertebral-body metastatic disease, with MR-thermometry subsequently applied to monitor the laser ablation process in real-time. ${ }^{71}$ The mainstay of nonsurgical oncologic treatment remains, of course, stereotactic body radiotherapy with either gamma-knife or linear accelerator-based radiosurgery. ${ }^{72}$ A comprehensive overview of radiosurgery is outside the scope of this review and will be left to the ample existing body of literature.

In more esoteric applications, transcutaneous pulsed laser stimulation has also been explored to counteract the degenerative changes of osteoporosis. In rodent models of ovariectomyand glucocorticoid-induced osteoporosis, transcutaneous laser stimulation over the posterior vertebral elements was found in isolation to have a detrimental effect on cortical and trabecular bone volume. However, when administered in conjunction with alendronate, an antiresorptive medication commonly prescribed to patients with osteoporosis, improvements in surrogate markers of bone health, including an increase in osteoblasts and type I collagen gene expression, and decrease in osteoclasts, were observed. $^{73}$

\subsection{Future Directions}

In the short term, future applications of optical techniques to spinal therapeutics may focus on extending current applications to the point where they may be considered superior to existing open surgical techniques. For instance, while PLD/PELD may result in equivalent short-term outcomes to open microdiscectomy, recurrence rates requiring subsequent open surgery remain unacceptably high. This may be due in part to the inability of current laser ablation techniques to resect bone adequately, and to be targeted appropriately, to perform a foraminotomy to indirectly decompress the affected nerve root, as is typically done in open microdiscectomy in addition to removing the offending disc fragments. Extension of current laser ablation techniques to safely and rapidly resect bone, as well as intervertebral ligaments, may allow percutaneous laser techniques to be extended to bilateral lumbar laminectomy/flavectomy for central stenosis, in addition to discectomy for unilateral radiculopathy as in the current paradigm. A typical open microdiscectomy can be performed safely and effectively in under $90 \mathrm{~min}$; adoption of laser or other optical ablation techniques therefore is predicated on ablation speeds permitting a total operation time equal or faster than this metric. One may imagine a scenario where, if laser bone ablation techniques become sufficiently advanced, facetectomies and osteotomies could be performed percutaneously via laser ablation in the context of long-segment deformity-correcting procedures, significantly reducing procedural morbidity and invasiveness. Advances in the application of light stimulation for bone formation may have multiple uses. Certainly, in the scenarios under current investigation, successful and efficacious transcutaneous application for patients with osteoporosis would represent a significant step forward in the treatment of this debilitating and economically burdensome disorder. Furthermore, in the context of spinal fusion procedures, one could conceive of percutaneous or open laser application following the placement of instrumentation, to stimulate local osteogenesis and therefore significantly accelerate osseous fusion, thereby reducing reoperation rates for malunion.

\section{Disclosures}

VXDY is cofounder of 7D Surgical Inc., a company licensing the OTI technology described in this article. There are no financial or other conflicts of interest arising from this role. The remaining authors have no conflicts of interest to declare.

\section{Acknowledgments}

Operating grants for VXDY have been provided by the Canada Research Council (CRC), Natural Sciences and Engineering Research Council of Canada (NSERC), and the Canada Foundation for Innovation (CFI). Salary support for DG has been provided by the Natural Sciences and Engineering Research Council of Canada (NSERC), Canadian Institutes of Health Research (CIHR), and the University of Toronto Surgeon-Scientist Training Program.

\section{References}

1. Y. Kuwazawa et al., "Biomechanical aspects of the cervical cord: effects of postural changes in healthy volunteers using positional magnetic resonance imaging," J. Spinal Disord. Tech. 19(5), 348-352 (2006).

2. C. P. Ames et al., "Cervical radiographical alignment," Spine 38, S149-S160 (2013).

3. R. A. Dickson, "Scoliosis: how big are you?" Orthopedics 10(6), 881-887 (1987).

4. P. Wessberg, B. I. Danielson, and J. Willén, "Comparison of Cobb angles in idiopathic scoliosis on standing radiographs and supine axially loaded MRI," Spine 31(26), 3039-3044 (2006).

5. H. Kanno et al., "Changes in lumbar spondylolisthesis on axial-loaded MRI: do they reproduce the positional changes in the degree of olisthesis observed on x-ray images in the standing position?" Spine J. 15(6), 1255-1262 (2015).

6. F. Alyas, D. Connell, and A. Saifuddin, "Upright positional MRI of the lumbar spine," Clin. Radiol. 63(9), 1035-1048 (2008).

7. N. J. Oxborrow, "Assessing the child with scoliosis: the role of surface topography," Arch. Dis. Child. 83(5), 453-455 (2000).

8. M. M. Doody et al., "Breast cancer following diagnostic x-rays among women with scoliosis," in Proc. 33rd Annual Meeting Scoliosis Research Society, New York, p. 104 (1998).

9. A. R. Levy et al., "Reducing the lifetime risk of cancer from spinal radiographs among people with adolescent idiopathic scoliosis," Spine 21(13), 1540-1547; discussion 1548 (1996).

10. M. M. Doody et al., "Breast cancer mortality after diagnostic radiography: findings from the U.S. Scoliosis Cohort Study," Spine 25(16), 2052-2063 (2000).

11. G. M. Ardran et al., "Assessment of scoliosis in children: low dose radiographic technique," Br. J. Radiol. 53(626), 146-147 (1980).

12. K. Abul-Kasim et al., "Low-dose helical computed tomography (CT) in the perioperative workup of adolescent idiopathic scoliosis," Eur. Radiol. 19(3), 610-618 (2009).

13. H. Takasaki, "Moiré topography," Appl. Opt. 9(6), 1467-1472 (1970).

14. F. Berryman et al., "A new system for measuring three-dimensional back shape in scoliosis," Eur. Spine J. 17(5), 663-672 (2008).

15. B. Drerup and E. Hierholzer, "Back shape measurement using video rasterstereography and three-dimensional reconstruction of spinal shape," Clin. Biomech. 9(1), 28-36 (1994).

16. C. J. Goldberg et al., "Surface topography, Cobb angles, and cosmetic change in scoliosis," Spine 26(4), E55-E63 (2001).

17. A. R. Turner-Smith, "A television/computer three-dimensional surface shape measurement system," J. Biomech. 21(6), 515-529 (1988).

18. S. Suzuki et al., "Ultrasound measurement of vertebral rotation in idiopathic scoliosis," J. Bone Jt. Surg. Br. 71(2), 252-255 (1989).

19. T. B. Grivas et al., "Study of trunk asymmetry in normal children and adolescents," Scoliosis 1(1), 19 (2006). 
20. T. Kotwicki et al., "Discrepancy in clinical versus radiological parameters describing deformity due to brace treatment for moderate idiopathic scoliosis," Scoliosis 2(1), 18 (2007).

21. F. Zaina, S. Negrini, and S. Atanasio, "TRACE (trunk aesthetic clinical evaluation), a routine clinical tool to evaluate aesthetics in scoliosis patients: development from the aesthetic index (AI) and repeatability," Scoliosis 4(1), 3 (2009).

22. I. H. Kalfas et al., "Application of frameless stereotaxy to pedicle screw fixation of the spine," J. Neurosurg. 83(4), 641-647 (1995).

23. J. P. Du et al., "Accuracy of pedicle screw insertion among 3 imageguided navigation systems: systematic review and meta-analysis," World Neurosurg. 109, 24-30 (2018).

24. A. Chan et al., "Intraoperative image guidance compared with free-hand methods in adolescent idiopathic scoliosis posterior spinal surgery: a systematic review on screw-related complications and breach rates," Spine J. 17, 1215-1229 (2017).

25. A. C. Bourgeois et al., "Improved accuracy of minimally invasive transpedicular screw placement in the lumbar spine with 3-dimensional stereotactic image guidance: a comparative meta-analysis," J. Spinal Disord. Tech. 28(9), 324-329 (2015).

26. N. Luther et al., "Comparison of navigated versus non-navigated pedicle screw placement in 260 patients and 1434 screws: screw accuracy, screw size, and the complexity of surgery," J. Spinal Disord. Tech. 28(5), E298-E303 (2015).

27. A. Mason et al., "The accuracy of pedicle screw placement using intraoperative image guidance systems," J. Neurosurg. Spine 20(2), 196-203 (2014).

28. N. F. Tian et al., "Pedicle screw insertion accuracy with different assisted methods: a systematic review and meta-analysis of comparative studies," Eur. Spine J. 20(6), 846-859 (2011).

29. B. J. Shin et al., "Pedicle screw navigation: a systematic review and meta-analysis of perforation risk for computer-navigated versus freehand insertion," J. Neurosurg. Spine 17(2), 113-122 (2012).

30. R. Verma et al., "Functional outcome of computer-assisted spinal pedicle screw placement: a systematic review and meta-analysis of 23 studies including 5,992 pedicle screws," Eur. Spine J. 19(3), 370-375 (2010).

31. I. D. Gelalis et al., "Accuracy of pedicle screw placement: a systematic review of prospective in vivo studies comparing free hand, fluoroscopy guidance and navigation techniques," Eur. Spine J. 21(2), 247-255 (2012).

32. L. P. Amiot et al., "Comparative results between conventional and computer-assisted pedicle screw installation in the thoracic, lumbar, and sacral spine," Spine 25(5), 606-614 (2000).

33. K. Izadpanah et al., "Computer navigation in balloon kyphoplasty reduces the intraoperative radiation exposure," Spine 34(12), 13251329 (2009).

34. S. Schafer et al., "Mobile C-arm cone-beam CT for guidance of spine surgery: image quality, radiation dose, and integration with interventional guidance," Med. Phys. 38(8), 4563-4574 (2011).

35. K. T. Foley, D. A. Simon, and Y. R. Rampersaud, "Virtual fluoroscopy: computer-assisted fluoroscopic navigation," Spine 26(4), 347-351 (2001).

36. K. G. Abdullah et al., "Radiation exposure to the spine surgeon in lumbar and thoracolumbar fusions with the use of an intraoperative computed tomographic 3-dimensional imaging system," Spine 37(17), E1074-E1078 (2012).

37. D. Mendelsohn et al., "Patient and surgeon radiation exposure during spinal instrumentation using intraoperative computed tomographybased navigation," Spine J. 16(3), 343-354 (2016).

38. J. R. Bandela et al., "Use of CT-based intraoperative spinal navigation: management of radiation exposure to operator, staff, and patients," World Neurosurg. 79(2), 390-394 (2013).

39. J. Lange et al., "Estimating the effective radiation dose imparted to patients by intraoperative cone-beam computed tomography in thoracolumbar spinal surgery," Spine 38(5), E306-E312 (2013).

40. R. Hartl et al., "Worldwide survey on the use of navigation in spine surgery," World Neurosurg. 79(1), 162-172 (2013).

41. Y.-H. Zhang et al., "Comparison perioperative factors during minimally invasive pre-psoas lateral interbody fusion of the lumbar spine using either navigation or conventional fluoroscopy," Global Spine J. 7(7), 657-663 (2017).
42. S. Rajasekaran et al., "Randomized clinical study to compare the accuracy of navigated and non-navigated thoracic pedicle screws in deformity correction surgeries," Spine 32(2), E56-E64 (2007).

43. E. Tabaraee et al., "Intraoperative cone beam-computed tomography with navigation (O-ARM) versus conventional fluoroscopy (C-ARM)," Spine 38(22), 1953-1958 (2013).

44. Y.-M. Ryang et al., "Learning curve of 3D fluoroscopy image-guided pedicle screw placement in the thoracolumbar spine," Spine J. 15(3), 467-476 (2015).

45. R. Khadem et al., "Comparative tracking error analysis of five different optical tracking systems," Comput. Aided Surg. 5(2), 98-107 (2000).

46. A. D. Wiles, D. G. Thompson, and D. D. Frantz, "Accuracy assessment and interpretation for optical tracking systems," Proc. SPIE 5367, 421-432 (2004).

47. A. Quiñones-Hinojosa et al., "Accuracy over space and time of computer-assisted fluoroscopic navigation in the lumbar spine in vivo," J. Spinal Disord. Tech. 19(2), 109-113 (2006).

48. C. DeLorenzo et al., "Image-guided intraoperative cortical deformation recovery using game theory: application to neocortical epilepsy surgery," IEEE Trans. Med. Imaging 29(2), 322-338 (2010).

49. S. Ji et al., "Patient registration using intraoperative stereovision in image-guided open spinal surgery," IEEE Trans. Biomed. Eng. 62(9), 2177-2186 (2015).

50. S. Ji et al., "Intraoperative CT as a registration benchmark for intervertebral motion compensation in image-guided open spinal surgery," Int. J. Comput. Assist. Radiol. Surg. 10(12), 2009-2020 (2015).

51. R. Jakubovic et al., "A.709: design and development of a novel, fast, extensive intraoperative registration technique of optical machine vision to pre-operative imaging for cranial and spinal neurosurgical procedures: clinical feasibility and comparison with existing neuronavi," J. Neurosurg. 124(4), A1146-A1209 (2016).

52. N. D. Glossop, "Advantages of optical compared with electromagnetic tracking," J. Bone Jt. Surg. Ser. A 91(Suppl. 1), 23-28 (2009).

53. A. Köpfle et al., "A modular scalable approach to occlusion-robust lowlatency optical tracking," Lect. Notes Comput. Sci. 3217, 1085-1086 (2004).

54. P. Paul, X. Morandi, and P. Jannin, "A surface registration method for quantification of intraoperative brain deformations in image-guided neurosurgery," IEEE Trans. Inf. Technol. Biomed. 13(6), 976-983 (2009).

55. H. Sun et al., "Stereopsis-guided brain shift compensation," IEEE Trans. Med. Imaging 24(8), 1039-1052 (2005).

56. A. D. Choo et al., "'Surgeons' perceptions of spinal navigation: analysis of key factors affecting the lack of adoption of spinal navigation technology," SAS J. 2(4), 189-194 (2008).

57. S. M. Presciutti, T. Karukanda, and M. Lee, "Management decisions for adolescent idiopathic scoliosis significantly affect patient radiation exposure," Spine J. 14(9), 1984-1990 (2014).

58. E. Ughwanogho et al., "Computed tomography-guided navigation of thoracic pedicle screws for adolescent idiopathic scoliosis results in more accurate placement and less screw removal," Spine 37(8), E473-E478 (2012).

59. J. Hsiang, "Wrong-level surgery: a unique problem in spine surgery," Surg. Neurol. Int. 2, 47 (2011).

60. W. Hsu et al., "Intraoperative localization of thoracic spine level with preoperative percutaneous placement of intravertebral polymethylmethacrylate," J. Spinal Disord. Tech. 21(1), 72-75 (2008).

61. M. G. Mody et al., "The prevalence of wrong level surgery among spine surgeons," Spine 33(2), 194-198 (2008).

62. N. M. Nasrabadi, "Pattern recognition and machine learning," $J$. Electron. Imaging 16(4), 049901 (2007).

63. S.-F. L. Lo et al., "Automatic localization of target vertebrae in spine surgery: clinical evaluation of the LevelCheck registration algorithm," Spine 40(8), E476-E483 (2015).

64. S. Erkan et al., "Alignment of pedicle screws with pilot holes: can tapping improve screw trajectory in thoracic spines?" Eur. Spine J. 19(1), 71-77 (2010).

65. M. F. Mínguez et al., "Quantifier variables of the back surface deformity obtained with a noninvasive structured light method: evaluation of their usefulness in idiopathic scoliosis diagnosis," Eur. Spine J. 16(1), 73-82 (2007). 
66. N. Moussazadeh et al., "Separation surgery for spinal metastases: effect of spinal radiosurgery on surgical treatment goals," Cancer Control 21(2), 168-174 (2014).

67. S. I. Tafazal and P. J. Sell, "Incidental durotomy in lumbar spine surgery: incidence and management," Eur. Spine J. 14(3), 287-290 (2005).

68. M. R. McMillan et al., "Percutaneous laser disc decompression," Photomed. Laser Surg. 25(1), 59-60 (2007).

69. P. A. Brouwer et al., "Percutaneous laser disc decompression versus conventional microdiscectomy for patients with sciatica: two-year results of a randomised controlled trial," Interventional Neuroradiol. 23(3), 313-324 (2017).

70. M. T. N. Knight, A. Goswami, and J. T. Patko, "Cervical percutaneous laser disc decompression: preliminary results of an ongoing prospective outcome study," J. Clin. Laser Med. Surg. 19(1), 3-8 (2001).

71. C. E. Tatsui et al., "Utilization of laser interstitial thermotherapy guided by real-time thermal MRI as an alternative to separation surgery in the management of spinal metastasis," J. Neurosurg. Spine 23(4), 400-411 (2015).

72. M. Huo et al., "Stereotactic spine radiosurgery: review of safety and efficacy with respect to dose and fractionation," Surg. Neurol. Int. 8, 30 (2017).
73. M. Fredoni et al., "Evaluation of the effects of photobiomodulation on vertebras in two rat models of experimental osteoporosis," Lasers Med. Sci. 32(7), 1545-1560 (2017).

Daipayan Guha completed three years of an honors BSc in Laboratory Medicine and Pathobiology at the University of Toronto, when he gained acceptance to the MD program, completed in 2012. He then entered neurosurgery residency training in Toronto, where he is currently a fifth-year resident. Following his third year, he entered the Surgeon-Scientist Program, completing a PhD in biomedical engineering under the supervision of Dr. Victor Yang. His research focus has been on image-guided spinal surgery, with his thesis work focusing on the feasibility of optical topographic imaging for spinal navigation.

Victor X. D. Yang received his Honors BASc in engineering science from the University of Toronto in 1997. He completed a master's in electrical and computer engineering in 1998, then entered the University of Toronto MD-PhD program in the Department of Medical Biophysics, completed in 2004. Currently, he is an associate professor in the Division of Neurosurgery, University of Toronto, and Department of Electrical and Computer Engineering, Ryerson University, holding the Canada Research Chair (CRC) in Bioengineering and Biophotonics. 